\title{
Interplay between genetic predisposition, macronutrient intake and type 2 diabetes incidence: analysis within EPIC-InterAct across eight European countries
}

\author{
Sherly X. $\mathrm{Li}^{1}$ • Fumiaki Imamura ${ }^{1}$ - Matthias B. Schulze ${ }^{2,3}$ • Jusheng Zheng ${ }^{1} \cdot$ Zheng Ye $^{1,4} \cdot$ Antonio Agudo $^{5}$. \\ Eva Ardanaz ${ }^{6,7}$. Dagfinn Aune ${ }^{8,9} \cdot$ Heiner Boeing ${ }^{10}$. Miren Dorronsoro ${ }^{7,11,12}$. Courtney Dow ${ }^{13} \cdot$ Guy Fagherazzi $^{13}$. \\ Sara Grioni ${ }^{14}$. Marc J. Gunter ${ }^{15}$. José María Huertaa ${ }^{7,16}$. Daniel B. Ibsen ${ }^{17}$. Marianne Uhre Jakobsen ${ }^{17,18}$. \\ Rudolf Kaaks ${ }^{19}$ - Timothy J. Key ${ }^{20}$ - Kay-Tee Khaw ${ }^{21}$. Cecilie Kyrø ${ }^{22}$ • Francesca Romana Mancini ${ }^{13}$. \\ Elena Molina-Portillo ${ }^{7,23} \cdot$ Neil Murphy ${ }^{15} \cdot$ Peter M. Nilsson ${ }^{24} \cdot$ N. Charlotte Onland-Moret ${ }^{25} \cdot$ Domenico Palli $^{26}$. \\ Salvatore Panico ${ }^{27}$. Alaitz Poveda ${ }^{28,29}$. J. Ramón Quirós ${ }^{30}$ • Fulvio Ricceri ${ }^{31,32}$ • Ivonne Sluijs ${ }^{25}$. \\ Annemieke M. W. Spijkerman ${ }^{33}$ • Anne Tjonneland ${ }^{34} \cdot$ Rosario Tumino $^{35,36}$ - Anna Winkvist ${ }^{37}$ • Claudia Langenberg ${ }^{1}$. \\ Stephen J. Sharp ${ }^{1} \cdot$ Elio Riboli $^{38} \cdot$ Robert A. Scott $^{1} \cdot$ Nita G. Forouhi $^{1} \cdot$ Nicholas J. Wareham $^{1}$
}

Received: 27 October 2017 / Accepted: 8 February 2018 / Published online: 17 March 2018

(C) The Author(s) 2018. This article is an open access publication

\begin{abstract}
Aims/hypothesis Gene-macronutrient interactions may contribute to the development of type 2 diabetes but research evidence to date is inconclusive. We aimed to increase our understanding of the aetiology of type 2 diabetes by investigating potential interactions between genes and macronutrient intake and their association with the incidence of type 2 diabetes.

Methods We investigated the influence of interactions between genetic risk scores (GRSs) for type 2 diabetes, insulin resistance and BMI and macronutrient intake on the development of type 2 diabetes in the European Prospective Investigation into Cancer and Nutrition (EPIC)-InterAct, a prospective case-cohort study across eight European countries ( $N=21,900$ with 9742 incident type 2 diabetes cases). Macronutrient intake was estimated from diets reported in questionnaires, including proportion of energy derived from total carbohydrate, protein, fat, plant and animal protein, saturated, monounsaturated and polyunsaturated fat and dietary fibre. Using multivariable-adjusted Cox regression, we estimated country-specific interaction results on the multiplicative scale, using random-effects meta-analysis. Secondary analysis used isocaloric macronutrient substitution.

Results No interactions were identified between any of the three GRSs and any macronutrient intake, with low-to-moderate heterogeneity between countries $\left(I^{2}\right.$ range $\left.0-51.6 \%\right)$. Results were similar using isocaloric macronutrient substitution analyses and when weighted and unweighted GRSs and individual SNPs were examined.

Conclusions/interpretation Genetic susceptibility to type 2 diabetes, insulin resistance and BMI did not modify the association between macronutrient intake and incident type 2 diabetes. This suggests that macronutrient intake recommendations to prevent type 2 diabetes do not need to account for differences in genetic predisposition to these three metabolic conditions.
\end{abstract}

Keywords BMI · Body mass index · Diabetes · Diet · Dietary fibre · Genetic risk score · GRS · Insulin resistance · Interaction · Macronutrient

Nita G. Forouhi and Nicholas J. Wareham are joint senior authors.

Electronic supplementary material The online version of this article (https://doi.org/10.1007/s00125-018-4586-2) contains peer-reviewed but unedited supplementary material, which is available to authorised users.

Nita G. Forouhi

nita.forouhi@mrc-epid.cam.ac.uk

Nicholas J. Wareham

nick.wareham@mrc-epid.cam.ac.uk

Extended author information available on the last page of the article

\section{Abbreviations}

EPIC European Prospective Investigation into Cancer and Nutrition

GRS Genetic risk score

\section{Introduction}

Genetic and environmental factors, including diet, contribute to the development of type 2 diabetes. Among dietary 


\section{Research in context}

\section{What is already known about this subject?}

- Type 2 diabetes is thought to be the result of the interplay between genes and the environment

- There is limited evidence on whether genetic predisposition to metabolic disease leads to differing response of macronutrient consumption on the risk of developing type 2 diabetes

\section{What is the key question?}

- Are there any gene-macronutrient interactions in the aetiology of type 2 diabetes, using overall genetic risk scores for obesity, insulin resistance and type 2 diabetes?

\section{What are the new findings?}

- We did not identify any statistically significant interactions to suggest that the specific examined interactions play a role in the development of type 2 diabetes

\section{How might this impact on clinical practice in the foreseeable future?}

- If interactions are identified and confirmed in the future, it may suggest that dietary interventions could be more efficacious if tailored according to someone's genetic profile, but current findings do not yet support this

components, an emphasis on macronutrient composition has dominated public health dietary recommendations for decades, with guidance on the optimal per cent of energy to be consumed from carbohydrate, fat and protein. More recent dietary guidance also acknowledges the importance of macronutrient quality. For instance, evidence supporting the cardiometabolic benefits of replacing dietary saturated fat with polyunsaturated fat has led to guidance concerning fat subtype or quality. There is also a substantial genetic contribution to type 2 diabetes, with the heritability estimated to be $40-80 \%$.

There has been increasing interest in whether this genetic susceptibility may influence how macronutrient intake affects the development of type 2 diabetes (gene-macronutrient interaction) and whether this may support the notion of 'personalised' or 'precision' nutrition. However, our recent systematic review failed to confirm any interactions via replication using similar cohorts [1]. Genetic risk scores (GRSs) may help to explain more variance for type 2 diabetes and prove better than candidate gene approaches to improve statistical power to detect potential interactions. Yet, there is a paucity of studies examining gene-macronutrient interaction using a GRS approach. Therefore, we aimed to increase our understanding of the aetiology of type 2 diabetes by investigating potential interactions between genes and macronutrient intake and their association with the incidence of type 2 diabetes using GRSs for type 2 diabetes, insulin resistance and BMI.

\section{Methods}

Study population and case definition and ascertainment EPIC-InterAct is a case-cohort study, nested within the
Fig. 1 Association between macronutrient intake and the incidence of type 2 diabetes (T2D) stratified by high or low GRS for T2D (a), insulin resistance (b) and BMI (c): EPIC-InterAct study. GRS categorisation: T2D high $\geq 52$, low $<52$ risk alleles; insulin resistance high $\geq 55$, low $<55$ risk alleles; BMI high $\geq 91$, low $<91$ risk alleles. Macronutrients are modelled per SD difference in intake (see Table 1 for the SD for each macronutrient). Carbohydrate intake adjusted for age (underlying time scale), sex, centre, education, physical activity, smoking status, sex-specific alcohol category, BMI, total energy intake, dietary protein, PUFA:SFA ratio, dietary fibre and first five principal components for population stratification. Intake of protein and its subtypes adjusted for age (underlying time scale), sex, centre, physical activity, smoking status, sex-specific alcohol categories, BMI, waist-hip ratio, total energy intake, dietary fibre, SFA, MUFA, PUFA, soft drinks, tea and coffee (not adjusted for carbohydrates [i.e. a substitution model]), education and first five principal components for population stratification. Intake of fat and its subtypes adjusted for age (underlying time scale), sex, centre, physical activity, smoking status, sex-specific alcohol categories, BMI, total energy intake, dietary fibre, magnesium, iron, vitamin C, leafy vegetables, tea, coffee, education and first five principal components for population stratification. Intake of dietary fibre and its subtypes adjusted for age (underlying time scale), sex, centre, physical activity, smoking status, sex-specific alcohol category, total energy intake, dietary carbohydrates, magnesium, SFA, education level and first five principal components for population stratification. Fibre subtypes were mutually adjusted. The interaction analysis for BMI GRS does not adjust for BMI. Interactions were considered statistically significant if $p<0.0015(0.05 /$ 33 tests). Example of interpretation: the HR of $1 \mathrm{SD}$ difference in fruit fibre on incident T2D is 1.03 in those who have the highest genetic predisposition for T2D and 1.01 for those with lower genetic predisposition for T2D. There was no statistically significant difference between those with different genetic predispositions for T2D. Black circles, high GRS; white circles, low GRS. MUFA, monounsaturated fatty acid; PUFA, polyunsaturated fatty acid; SFA, saturated fatty acid 
a

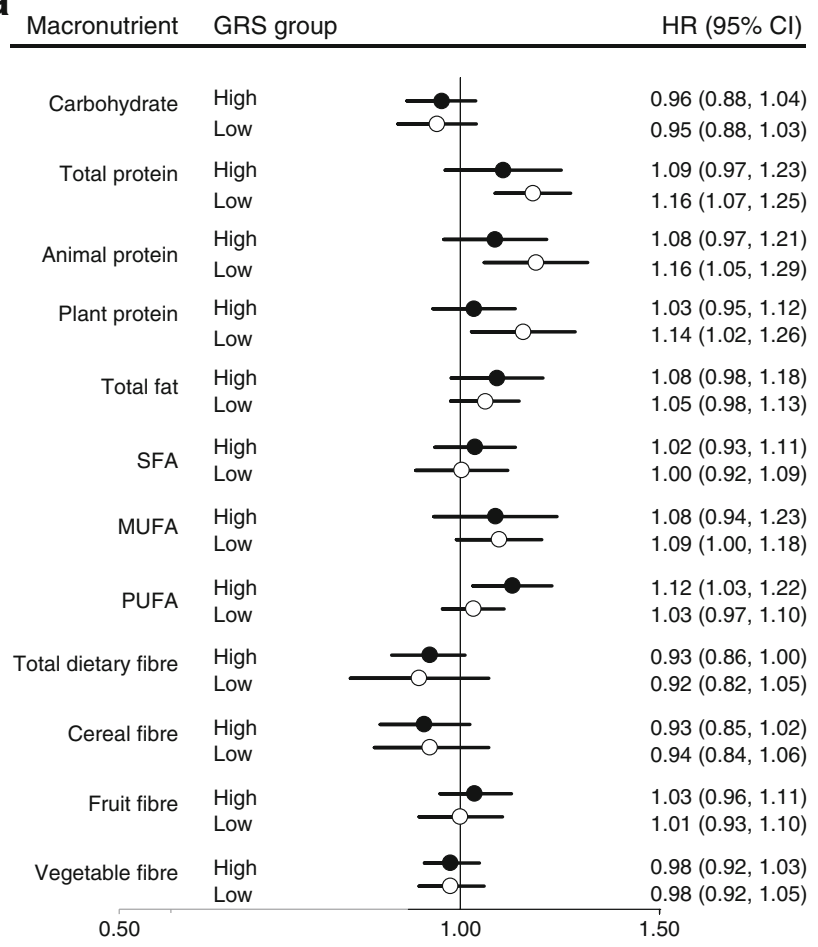

HR for incident T2D, per SD of the respective macronutrients b

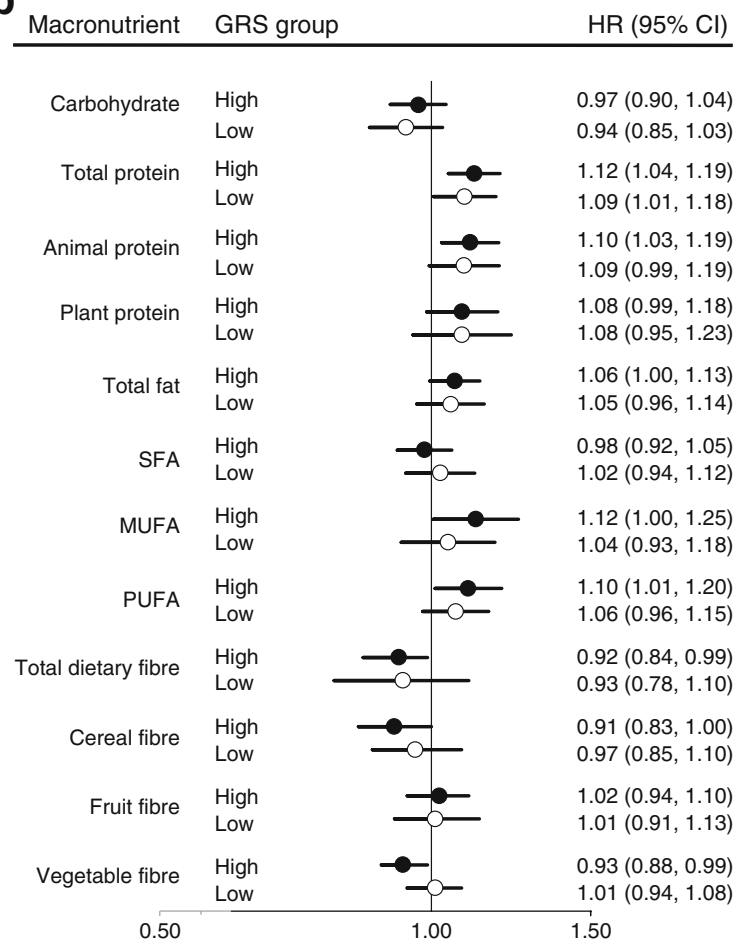

HR for incident T2D, per SD of the respective macronutrients

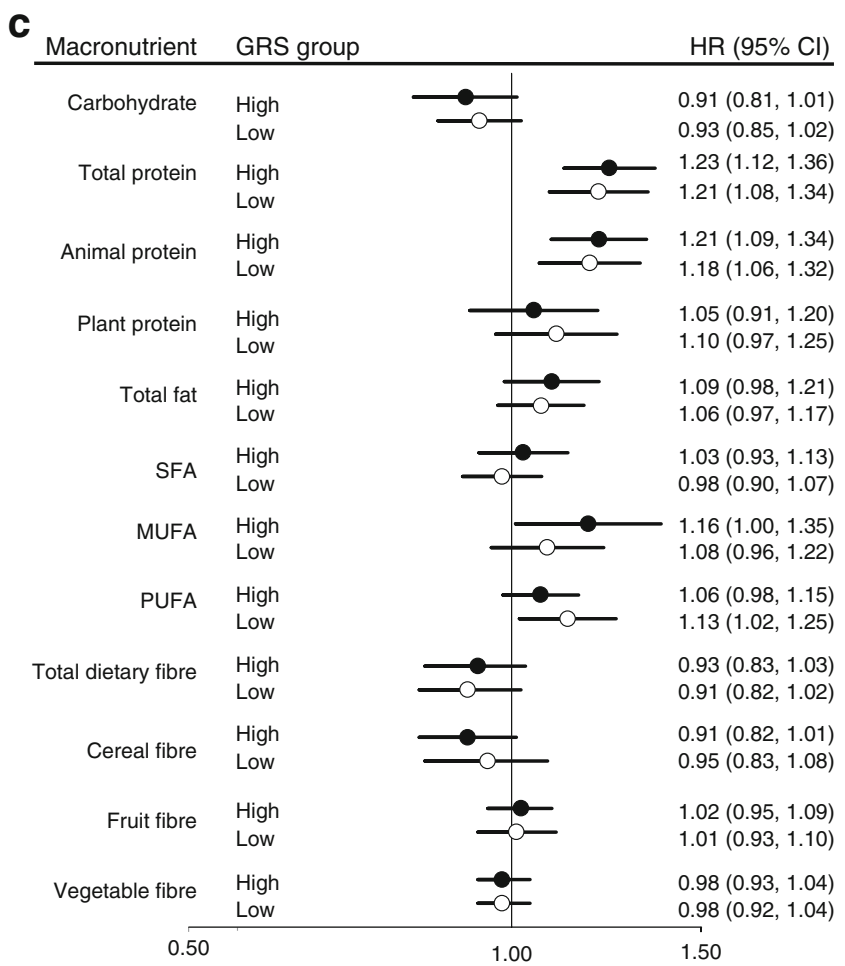

HR for incident T2D, per SD of the respective macronutrients

European Prospective Investigation into Cancer and Nutrition (EPIC) study, described previously [2]. From 340,234 eligible participants across eight European countries, EPIC-InterAct included 27,779 healthy participants, consisting of 12,403 incident type 2 diabetes cases and a representative subcohort of 16,154 participants (including 778 individuals who developed type 2 diabetes during follow-up, according to the design of a case-cohort study). 
Cases of type 2 diabetes were ascertained via self-report of a diagnosis by a medical doctor or use of glucose-lowering medication noted in a lifestyle questionnaire and verified by one or more independent sources (linkage to primary and secondary care registers, medication use from prescription registers, hospital admission, mortality data and individual medical record review in some centres). The study period was from baseline (1991-1997) until the censor date of 31 December 2007. Our current analyses were based on 21,900 adults with available genome-wide genotyping and dietary data (electronic supplementary material [ESM] Fig. 1). Participants gave written informed consent and ethical approval was obtained at each participating research centre.

Exposure and covariates Genotyping was performed on the Illumina 660 W-Quad BeadChip (http://emea.support. illumina.com/array/array_kits/human660w-quad_dna_ analysis_kit.html) or Illumina HumanCore Exome chip (http://emea.support.illumina.com/array/array_kits/ humancore_exome_beadchip_kit.html) arrays, with imputation to the Haplotype Reference Consortium using IMPUTE v2.3.2 (http://mathgen.stats.ox.ac.uk/impute/ impute_v2.html). All SNPs met quality control criteria for genotyping call rate $(\geq 95 \%)$ or were well imputed (info $\geq 0.99$ ). We generated unweighted GRSs for type 2 diabetes, insulin resistance and BMI by summing up the number of risk alleles for each trait using SNPs that reached genome-wide significance for the respective traits in published meta-analyses investigating European populations [3-5]. Habitual self-reported macronutrient intakes were estimated from country-specific baseline dietary assessments and food composition derived from the EPIC Nutrient DataBase. We examined macronutrient quantity (total carbohydrate, fat and protein intake) and quality (dietary fibre, saturated, monounsaturated and polyunsaturated fatty acids, and animal and plant protein).

Statistical analysis Variables with $<30 \%$ missing data were imputed using multiple imputation by chained equations in Stata (v14 [StataCorp, College Station, TX, USA]) (ESM Table 1). After confirming no obvious between-imputation variation across 20 multiple imputation datasets, a single imputation was used for analyses because of computational efficiency (ESM Fig. 2). Exposures were treated as continuous variables (GRS per SD difference and macronutrient densities as $5 \%$ of total energy intake per day and $1 \mathrm{~g} / 4.18 \mathrm{MJ}$ [or per $1000 \mathrm{kcal}$ ] per day for dietary fibre) to maximise statistical power. Crude and multivariable-adjusted Prentice-weighted Cox regression models were constructed within country (for macronutrient main associations) and by genotyping chip (for GRS main associations and gene--macronutrient interactions). Given the over-representation of cases in the case-cohort analysis, the cases within and outside the subcohort were weighted differently using the weighting scheme proposed by Prentice [6]. Country-specific HRs for the variables of interest were combined across countries using random-effects meta-analysis and, where appropriate, meta-analysed across genotyping chip. Multiplicative interaction was evaluated by fitting a product term between the genetic and macronutrient exposures. For consistency, modelling was based as closely as possible on the models used in previous EPIC-InterAct analyses for carbohydrate [7], protein [8] and dietary fibre [9] (ESM Methods). Between-country heterogeneity was quantified by the $I^{2}$ value and $p$ for heterogeneity was derived from the Cochran-Q test.

Further secondary interaction analysis was conducted for each SNP within all three GRSs. We also examined the effect of isocaloric macronutrient substitution on these interactions using the multivariate nutrient density model (ESM Table 2).

For visualisation, we also estimated the HR for each dietary factor stratified by high and low GRS groups (Fig. 1).

Stata v14 was used for analysis. Numerical $p$ values for interaction were reported; however, the threshold for determining statistical significance for interactions between GRS and macronutrient intake was $\leq 0.0015$ (0.05/33 tests) to account for the effective number of independent tests among correlated exposures (ESM Table 3).

\section{Results}

Table 1 shows the baseline characteristics, with more detail previously published [9], and main associations for macronutrient intake and GRSs. Positive associations with incident type 2 diabetes were observed for the proportion of energy from overall protein and animal protein intake (Table 1). However, these associations were not significant after accounting for multiple testing. No statistically significant interactions were identified-the association between the proportion of energy derived from the intake of each macronutrient and incident type 2 diabetes did not differ significantly by GRS for type 2 diabetes ( $p_{\text {interaction }} \geq 0.20$ ), insulin resistance $\left(p_{\text {interaction }} \geq 0.21\right)$ or BMI $\left(p_{\text {interaction }} \geq 0.22\right)$ (Fig. 1 and ESM Table 4). There was low-to-moderate heterogeneity between countries in EPIC-InterAct $\left(I^{2}\right.$ range $\left.0-51.6 \%\right)$ (ESM Table 4).

Secondary analysis Results did not change substantially when: (1) using weighted GRSs; (2) modelling isocaloric macronutrient substitution $\left(p_{\text {interaction }} \geq 0.17\right)$ (see model 5 in ESM Table 2); or (3) when examining interactions with each individual SNP while accounting for isocaloric macronutrient substitution (ESM Fig. 3 and ESM Table 5). The results were similar when our current analyses based on imputed data were compared 
Table 1 Main association between macronutrient intake or GRS and incidence of type 2 diabetes: EPIC-InterAct study

\begin{tabular}{|c|c|c|c|c|}
\hline Variable & No. cases/total & Subcohort non-cases & Total incident $\mathrm{T} 2 \mathrm{D}$ cases & $\mathrm{HR}(95 \% \mathrm{CI})$ per $\mathrm{SD}^{\mathrm{a}}$ \\
\hline Median follow-up, years & $9742 / 21,900$ & 12.3 & 6.8 & \\
\hline Age at baseline, years & & $52.3(9.3)$ & $55.7(7.6)$ & \\
\hline Sex, $\%$ male & & 37.9 & 49.9 & \\
\hline \multicolumn{5}{|l|}{ Macronutrient intake } \\
\hline Carbohydrate, \% TEI & $9742 / 21,900$ & $44.1(6.9)$ & $43.7(6.9)$ & $0.97(0.92,1.02)$ \\
\hline Protein, \% TEI & $9742 / 21,900$ & $16.9(3.0)$ & $17.2(3.0)$ & $1.10(1.03,1.18)$ \\
\hline Animal protein, $\%$ TEI & $9742 / 21,900$ & $10.5(3.2)$ & $10.9(3.2)$ & $1.10(1.01,1.18)$ \\
\hline Plant protein, \% TEI & $9742 / 21,900$ & $5.0(1.3)$ & $4.9(1.3)$ & $1.074(0.999,1.150)$ \\
\hline Fat, \% TEI & $9742 / 21,900$ & $34.8(5.7)$ & $34.7(5.7)$ & $1.03(0.99,1.08)$ \\
\hline SFA, \% TEI & $9742 / 21,900$ & $13.4(3.3)$ & $13.3(3.3)$ & $0.99(0.93,1.06)$ \\
\hline MUFA, \% TEI & $9742 / 21,900$ & $13.1(3.4)$ & $13.0(3.4)$ & $1.04(0.97,1.12)$ \\
\hline PUFA, \% TEI & $9742 / 21,900$ & $5.5(1.8)$ & $5.6(1.8)$ & $1.066(0.999,1.137)$ \\
\hline Fibre, $g$ & $9742 / 21,900$ & $22.7(7.5)$ & $22.6(7.6)$ & $0.92(0.84,1.02)$ \\
\hline Cereal, g & $9739 / 21,891$ & $8.8(4.9)$ & $8.9(4.9)$ & $0.96(0.86,1.07)$ \\
\hline Fruit, g & $9608 / 21,611$ & $4.3(3.2)$ & $4.2(3.2)$ & $0.86(0.73,1.02)$ \\
\hline Vegetable, $g$ & $9737 / 21,893$ & $4.1(2.6)$ & $34.0(2.6)$ & $0.99(0.94,1.04)$ \\
\hline \multicolumn{5}{|l|}{ GRS } \\
\hline $\mathrm{T} 2 \mathrm{D}$ (per 4.3 risk alleles) & - & - & - & $1.49(1.37,1.63)$ \\
\hline IR (per 4.5 risk alleles) & - & - & - & $1.14(1.09,1.20)$ \\
\hline BMI (per 6.3 risk alleles) & - & - & - & $1.07(1.04,1.10)^{\mathrm{b}}$ \\
\hline
\end{tabular}

Data are means (SD) unless stated otherwise

HRs for macronutrients (per SD) and incident T2D: carbohydrate intake adjusted for age (underlying time scale), sex, centre, education, physical activity, smoking status, sex-specific alcohol category, BMI, TEI, dietary protein, PUFA:SFA ratio, dietary fibre (attempt to replicate model 3 in Sluijs et al [7]); intake of protein and its subtypes adjusted for age (underlying time scale), sex, centre, physical activity, smoking status, sex-specific alcohol category, BMI, waist-hip ratio, TEI, dietary fibre, SFA, MUFA, PUFA, soft drinks, tea and coffee (not adjusted for carbohydrates; i.e. a substitution model), education (attempt to replicate model 4 in van Nielen et al [8]); intake of fat and its subtypes adjusted for age (underlying time scale), sex, centre, physical activity, smoking status, sex-specific alcohol category, BMI, TEI, dietary fibre, magnesium, iron, vitamin C, leafy vegetables, tea, coffee, education; intake of dietary fibre and its subtypes adjusted for age (underlying time scale), sex, centre, physical activity, smoking status, sex-specific alcohol category, TEI, dietary carbohydrates, magnesium, saturated fatty acids, education level. Fibre subtypes were mutually adjusted (attempt to replicate model 3 in The InterAct Consortium, 2015 [9]). HR for GRSs and T2D: adjusted for age (underlying time scale), sex, centre, first five principal components for population stratification and BMI. No. of SNPs: T2D 48 (as per Morris et al [3]), BMI 97 (as per Locke et al [4]), IR 53 (as per Lotta et al [5])

${ }^{\mathrm{a}} \mathrm{SD}$ calculated based on the whole population

${ }^{\mathrm{b}}$ BMI GRS does not include adjustment for BMI

IR, insulin resistance; MUFA, monounsaturated fatty acid; PUFA, polyunsaturated fatty acid; SFA, saturated fatty acid; T2D, type 2 diabetes; TEI, total energy intake

with a complete case analysis (ESM Table 6 provides an example).

\section{Discussion}

In this large, multi-country, population-based prospective study from Europe, we found no statistically significant interactions between three metabolic GRSs and macronutrient intake on the development of type 2 diabetes. All three GRSs were positively associated with incident type 2 diabetes [3-5] and the associations between macronutrient intake and type 2 diabetes were directionally consistent with previous literature (Table 1) [7-9].
The literature on gene-macronutrient interaction studies and type 2 diabetes, using a GRS, is limited. A crosssectional study which examined the interaction between a type 2 diabetes GRS and carbohydrate and fibre intake failed to identify interactions for prevalent type 2 diabetes $(N=1337$ cases of type 2 diabetes) [10]. Our work is the first to examine gene-macronutrient interactions for type 2 diabetes risk prospectively using three GRSs, comprehensively investigating all major macronutrients, and consists of a large sample $(N=$ 9742 cases of type 2 diabetes). The consistency across various methods (adoption of unweighted and weighted GRSs, combined GRSs as well as their constituent SNPs and application of isocaloric macronutrient substitution modelling) collectively strengthens the confidence in our null findings for interaction. 
There are several factors that may contribute to the absence of interactions in our current study. Other dietary exposures, such as foods and/or dietary patterns, may offer greater insight compared with nutrients based on the food synergism hypothesis and may be subject to less accumulated measurement error. There may also be other genetic loci, with no or weak marginal genetic effects for our traits of interest, that may show a significant variation in effect between subgroups of the population. A GRS may mask interactions with individual SNPs and so may reduce statistical efficiency. Therefore, we also examined individual SNP interactions but did not identify any that were statistically significant. The generalisability of our findings is limited to European populations and research is warranted in other populations.

Among this study's strengths, EPIC-InterAct's prospective design minimises the potential bias due to recall bias and reverse causality for dietary exposures and the verification of diabetes cases minimises possible misclassification bias of the outcome. To our knowledge, this study represents the most comprehensive investigation of the interaction between multiple GRSs and macronutrient intake on incident type 2 diabetes, to date. We tried to address some of the key methodological issues identified from our recent systematic review, including multiple testing and inadequate control for likely confounders [1]. To reduce the risk of spurious gene-macronutrient interactions, we confirmed that the GRSs were not correlated with macronutrient intake. To our knowledge, this is also the first observational study of gene-macronutrient interactions within the cardiometabolic literature that has investigated the effect of isocaloric macronutrient substitution, which is important for public health interpretation of macronutrient density.

In conclusion, within a multi-centre European cohort, we observed no interaction between GRSs for type 2 diabetes, insulin resistance and BMI and macronutrient intake on the risk for developing type 2 diabetes. These findings suggest that currently there is no support for personalised dietary advice on macronutrient intake for type 2 diabetes prevention in subgroups of the population defined by their overall genetic risk for type 2 diabetes, insulin resistance or BMI.

Acknowledgements We thank all participants and staff who contributed to the study within their respective EPIC centres. We thank N. Kerrison of the Medical Research Council (MRC) Epidemiology Unit, Cambridge, UK for managing the data for the InterAct project. We thank staff from the Technical, Field Epidemiology and Data Functional Group Teams of the MRC Epidemiology Unit in Cambridge for carrying out sample preparation, DNA provision and quality control, genotyping and data-handling work. S.X. Li thanks the Commonwealth Scholarship Commission and the Cambridge Trust for supporting her PhD studies.

Data availability Researchers seeking the analysis dataset for this work can submit a data request to the EPIC-InterAct study central contact point by emailing interact@mrc-epid.cam.ac.uk.
Funding The InterAct project is supported by the EU Sixth Framework Programme (FP6) (grant LSHM_CT_2006_037197). The InterAct investigators acknowledge support from the Medical Research Council Epidemiology Unit (grants MC_UU_12015/1 and MC_UU_12015/5), Medical Research Council Human Nutrition Research (grant MC UP A090_1006) and Cambridge Lipidomics Biomarker Research Initiative (grant G0800783). NJW and NGF acknowledge IS-BRC-1215-20014 funding support from NIHR Biomedical Research Centre Cambridge. MBS was supported by a grant from the German Federal Ministry of Education and Research (BMBF) to the German Center for Diabetes Research (DZD) and the State of Brandenburg. Support was also provided by the following bodies: Health Research Fund (FIS) of the Spanish Ministry of Health and Navarre Regional Government (to EA); Health Research Fund of the Spanish Ministry of Health and Murcia Regional Government (No. 6236) (to JMH); German Cancer Aid, German Ministry of Research (BMBF) (to RK); Cancer Research UK C8221/ A19170 and Medical Research Council MR/M012190/1 (to TJK); Medical Research Council UK and Cancer Research UK (to KTK); Swedish Research Council (to PMN); Dutch Ministry of Public Health, Welfare and Sports (VWS), Netherlands Cancer Registry (NKR), LK Research Funds, Dutch Prevention Funds, Dutch ZON (Zorg Onderzoek Nederland), World Cancer Research Fund (WCRF) and Statistics Netherlands (to NCOM, IS, AMWS and EPIC Bilthoven and Utrecht); Compagnia di San Paolo (to SP); Regional Government of Asturias (to JRQ); Danish Cancer Society (to AT); Sicilian Regional Government and AIRE-ONLUS Ragusa (to RT and EPIC Ragusa) and Imperial College Biomedical Research Centre (to ER). Verification of diabetes cases in EPIC-NL was additionally funded by NL Agency grant IGE05012 and an Incentive Grant from the Board of the UMC Utrecht.

Duality of interest The authors declare that there is no duality of interest associated with this manuscript.

Contribution statement SXL and RAS had full access to all of the data in the study and take responsibility for the integrity of the data and the accuracy of the data analysis. SXL performed the statistical analyses and wrote the first draft with supervision from NGF and NJW. NJW, CL, NGF and SJS coordinated the InterAct project. The Working Group (RAS, FI, MBS, JSZ, ZY) and all other authors contributed to interpretation of data, revised the article critically for important intellectual content and approved the final version of the manuscript.

Open Access This article is distributed under the terms of the Creative Commons Attribution 4.0 International License (http:// creativecommons.org/licenses/by/4.0/), which permits unrestricted use, distribution, and reproduction in any medium, provided you give appropriate credit to the original author(s) and the source, provide a link to the Creative Commons license, and indicate if changes were made.

\section{References}

1. Li SX, Imamura F, Ye Z et al (2017) Interaction between genes and macronutrient intake on the risk of developing type 2 diabetes: systematic review and findings from EPIC- InterAct. Am J Clin Nutr 106:263-275

2. Langenberg C, Sharp S, Forouhi NG et al (2011) Design and cohort description of the InterAct Project: an examination of the interaction of genetic and lifestyle factors on the incidence of type 2 diabetes in the EPIC Study. Diabetologia 54:2272-2282

3. Morris AP, Voight BF, Teslovich TM et al (2012) Large-scale association analysis provides insights into the genetic architecture and pathophysiology of type 2 diabetes. Nat Genet 44:981-990 
4. Locke AE, Kahali B, Berndt SI et al (2015) Genetic studies of body mass index yield new insights for obesity biology. Nature 518:197-206

5. Lotta L, Gulati P, Day FR et al (2016) Integrative genomic analysis implicates limited peripheral adipose storage capacity in the pathogenesis of human insulin resistance. Nat Genet 49:17-26

6. Barlow WE, Ichikawa L, Rosner D, Izumi S (1999) Analysis of case-cohort designs. J Clin Epidemiol 52:1165-1172

7. Sluijs I, Beulens JWJ, Van Der Schouw YT et al (2013) Dietary glycemic index, glycemic load, and digestible carbohydrate intake are not associated with risk of type 2 diabetes in eight European countries. J Nutr 143:93-99
8. van Nielen M, Feskens EJM, Mensink M et al (2014) Dietary protein intake and incidence of type 2 diabetes in Europe: the EPICInterAct Case-Cohort Study. Diabetes Care 37:1854-1862

9. The InterAct Consortium (2015) Dietary fibre and incidence of type 2 diabetes in eight European countries: the EPIC-InterAct Study and a meta-analysis of prospective studies. Diabetologia 58:1394-1408

10. Villegas R, Goodloe RJ, McClellan BE, Boston J, Crawford DC (2014) Gene-carbohydrate and gene-fiber interactions and type 2 diabetes in diverse populations from the National Health and Nutrition Examination Surveys (NHANES) as part of the epidemiologic architecture for genes linked to environment (EAGLE) study. BMC Genet 15:1-8

\section{Affiliations}

Sherly X. $\mathrm{Li}^{1}$ • Fumiaki Imamura ${ }^{1}$ - Matthias B. Schulze ${ }^{2,3}$ • Jusheng Zheng ${ }^{1} \cdot$ Zheng Ye $^{1,4} \cdot$ Antonio Agudo $^{5}$. Eva Ardanaz ${ }^{6,7}$. Dagfinn Aune ${ }^{8,9}$. Heiner Boeing ${ }^{10}$. Miren Dorronsoro ${ }^{7,11,12}$. Courtney Dow ${ }^{13}$. Guy Fagherazzi ${ }^{13}$. Sara Grioni ${ }^{14}$. Marc J. Gunter ${ }^{15}$. José María Huerta ${ }^{7,16}$. Daniel B. Ibsen ${ }^{17}$. Marianne Uhre Jakobsen ${ }^{17,18}$. Rudolf Kaaks ${ }^{19}$. Timothy J. Key ${ }^{20} \cdot$ Kay-Tee Khaw $^{21} \cdot$ Cecilie Kyrø ${ }^{22}$. Francesca Romana Mancini ${ }^{13}$. Elena Molina-Portillo ${ }^{7,23}$. Neil Murphy ${ }^{15} \cdot$ Peter M. Nilsson ${ }^{24} \cdot$ N. Charlotte Onland-Moret ${ }^{25} \cdot$ Domenico Palli $^{26}$. Salvatore Panico ${ }^{27}$ - Alaitz Poveda ${ }^{28,29}$ • J. Ramón Quirós ${ }^{30}$ • Fulvio Ricceri ${ }^{31,32}$ • Ivonne Sluijs ${ }^{25}$. Annemieke M. W. Spijkerman ${ }^{33}$ • Anne Tjonneland ${ }^{34} \cdot$ Rosario Tumino $^{35,36}$ - Anna Winkvist ${ }^{37}$ • Claudia Langenberg ${ }^{1}$. Stephen J. Sharp ${ }^{1}$ - Elio Riboli ${ }^{38} \cdot$ Robert A. Scott $^{1} \cdot$ Nita G. Forouhi $^{1} \cdot$ Nicholas J. Wareham $^{1}$

1 MRC Epidemiology Unit, University of Cambridge School of Clinical Medicine, Box 285 Institute of Metabolic Science, Cambridge Biomedical Campus, Cambridge CB2 0QQ, UK

2 Department of Molecular Epidemiology, German Institute of Human Nutrition Potsdam-Rehbruecke, Nuthetal, Germany

3 German Center for Diabetes Research (DZD), MünchenNeuherberg, Germany

4 Department of Medical Genetics, Cambridge Institute for Medical Research (CIMR), University of Cambridge, Cambridge, UK

5 Catalan Institute of Oncology (ICO), Barcelona, Spain

6 Navarre Public Health Institute (ISPN), Pamplona, Spain

7 CIBER de Epidemiología y Salud Pública (CIBERESP), Madrid, Spain

8 Department of Epidemiology and Biostatistics, School of Public Health, Imperial College London, London, UK

9 Bjørknes University College, Oslo, Norway

10 Department of Epidemiology, German Institute of Human Nutrition Potsdam-Rehbruecke, Nuthetal, Germany

11 Public Health Division of Gipuzkoa, San Sebastian, Spain

12 Instituto BIO-Donostia, Basque Government, San Sebastian, Spain

13 CESP, Faculty of Medicine, University Paris-South, Faculty of Medicine, University Versailles-St Quentin, Inserm U1018, University Paris-Saclay, Villejuif, France

14 Epidemiology and Prevention Unit, Fondazione IRCCS, Istituto Nazionale dei Tumori, Milan, Italy
15 International Agency for Research on Cancer, Lyon, France

16 Department of Epidemiology, Murcia Regional Health Council, IMIB-Arrixaca, Murcia, Spain

17 Department of Public Health, Section for Epidemiology, Aarhus University, Aarhus, Denmark

18 National Food Institute, Division for Diet, Disease Prevention and Toxicology, Technical University of Denmark, Kongens Lyngby, Denmark

19 Division of Cancer Epidemiology, German Cancer Research Center (DKFZ), Heidelberg, Germany

20 Cancer Epidemiology Unit, University of Oxford, Oxford, UK

21 Department of Public Health and Primary Care, University of Cambridge, Cambridge, UK

22 Danish Cancer Society, Copenhagen, Denmark

23 Andalusian School of Public Health, Hospitales Universitarios de Granada/Universidad de Granada, Granada, Spain

24 Department of History of Medicine, Lund University, Malmö, Sweden

25 Julius Center for Health Sciences and Primary Care, University Medical Center Utrecht, Utrecht, the Netherlands

26 Cancer Risk Factors and Life-Style Epidemiology Unit, Cancer Research and Prevention Institute - ISPO, Florence, Italy

27 Dipartimento di Medicina Clinica e Chirurgia, Federico II University, Naples, Italy

28 Department of Clinical Sciences, Clinical Research Center, Skåne University Hospital, Lund University, Malmö, Sweden 
29 Department of Nutritional Research, Umeå University, Umeå, Sweden

30 Public Health Directorate, Asturias, Spain

31 Department of Clinical and Biological Sciences, University of Turin, Turin, Italy

32 Unit of Epidemiology, Regional Health Service ASL TO3, Grugliasco, (TO), Italy

33 National Institute for Public Health and the Environment (RIVM), Bilthoven, the Netherlands
34

Danish Cancer Society Research Center, Copenhagen, Denmark

35 Azienda Sanitaria Provinciale di Ragusa (ASP), Ragusa, Italy

36 Associazione Iblea per la Ricerca Epidemiologica (AIRE-ONLUS), Ragusa, Italy

37 The Sahlgrenska Academy, Department of Internal Medicine and Clinical Nutrition, University of Gothenburg, Göteborg, Sweden

38 School of Public Health, Imperial College London, London, UK 